\title{
Association between hemodynamics, morphology, and rupture risk of intracranial aneurysms: a computational fluid modeling study
}

\author{
Tianlun Qiu ${ }^{1}$ Guoliang Jin ${ }^{1} \cdot$ Haiyan $\mathrm{Xing}^{2} \cdot$ Haitao $\mathrm{Lu}^{3}$
}

Received: 13 December 2016/ Accepted: 7 March 2017 / Published online: 11 March 2017

(c) The Author(s) 2017. This article is published with open access at Springerlink.com

\begin{abstract}
The objective of the study was to examine the correlations between intracranial aneurysm morphology and wall shear stress (WSS) to identify reliable predictors of rupture risk. Seventy-two intracranial aneurysms (41 ruptured and 31 unruptured) from 63 patients were studied retrospectively. All aneurysms were divided into two categories: narrow (aspect ratio $\geq 1.4$ ) and wide-necked (aspect ratio $<1.4$ or neck width $\geq 4 \mathrm{~mm}$ ). Computational fluid dynamics was used to determine the distribution of WSS, which was analyzed between different morphological groups and between ruptured and unruptured aneurysms. Sections of the walls of clipped aneurysms were stained with hematoxylin-eosin, observed under a microscope, and photographed. Ruptured aneurysms were statistically more likely to have a greater low WSS area ratio (LSAR) $(P=0.001)$ and higher aneurysms parent WSS ratio $(P=0.026)$ than unruptured aneurysms. Narrownecked aneurysms were statistically more likely to have a larger LSAR $(P<0.001)$ and lower values of MWSS $(P<0.001)$, mean aneurysm-parent WSS ratio $(P<0.001)$, HWSS $(P=0.012)$, and the highest aneurysm-parent WSS ratio $(P<0.001)$ than wide-necked
\end{abstract}

Electronic supplementary material The online version of this article (doi:10.1007/s10072-017-2904-y) contains supplementary material, which is available to authorized users.

Tianlun Qiu

sxrqt1@163.com; 3503340264@qq.com

1 Department of Neurosurgery, Shaoxing People's Hospital, Shaoxing 312000, Zhejiang, China

2 School of Medicine, Shaoxing University, Zhejiang, China

3 Department of Neurosurgery, Chongming Branch of Shanghai Xinghua Hospital, Chongming 202150, Shanghai, China aneurysms. The aneurysm wall showed two different pathological changes associated with high or low WSS in wide-necked aneurysms. Aneurysm morphology could affect the distribution and magnitude of WSS on the basis of differences in blood flow. Both high and low WSS could contribute to focal wall damage and rupture through different mechanisms associated with each morphological type.

Keywords Intracranial aneurysms · Wall shear stress · Hemodynamics · Computational fluid dynamics

\section{Introduction}

Aneurysm rupture is the most common cause of nontraumatic subarachnoid hemorrhage. Hemodynamics are recognized as some of the many factors responsible for aneurysm rupture [1]. Computational fluid dynamics (CFD) is a branch of fluid mechanics that uses numerical analysis and data structures to solve and analyze problems that involve fluid flows. Image-based computational fluid dynamics (CFD) modeling identified associations between the hemodynamics of intracranial aneurysms and the likelihood of their growth and rupture [2], highlighting the promising possibility that aneurysmal hemodynamics may provide objective metrics to improve the stratification of rupture risk [3], but the growing number of proposed hemodynamic parameters makes it difficult to establish a consensus on this issue [4-6].

Wall shear stress (WSS) means the tangential drag force produced by horizontal movement of the fluid across the surface. In blood vessel, WSS acts on endothelium and is the mechanical force responsible for the acute change in luminal diameter. Currently, conflicting results concerning 
aneurysmal WSS, the most frequently explored hemodynamic parameter of augmented rupture risk, puzzle the IA research and clinical communities. Both high and low aneurysmal WSS are correlated with intracranial aneurysm growth and rupture [5], but disparate findings typically fall into four categories: (1) high spatial mean WSS (MWSS) is associated with rupture [7]; (2) low spatial MWSS is associated with rupture [2, 8, 9]; (3) high maximum WSS is associated with rupture [10,11]; and (4) WSS is not associated with rupture [12-14].

Valencia et al. [15] observed an association between the mean WSS at the aneurysmal sac and aneurysm surface index in lateral unruptured and ruptured aneurysms. 4Dflow magnetic resonance imaging was used to demonstrate that large/giant saccular aneurysms and small saccular aneurysms have higher peak velocities and WSS compared with fusiform aneurysms [16]. WSS was increased in giant saccular aneurysms, indicating a relationship between aneurysm size and hemodynamics. There is an increase in aneurysm area exposed to low WSS once the aneurysm size ratio (ASR) is decreased and the flow became slower [17].

With the above discrepancies and lack of consensus in mind, the objective of this study was to analyze whether WSS was significantly different in ruptured and unruptured aneurysms, whether WSS was significantly different in aneurysms with two disparate aspect ratios, and whether the hemodynamics are significantly different between the two groups.

\section{Materials and methods}

\section{Study design and patients}

This was a retrospective study of the imaging data of 72 aneurysms in 63 patients from the hospital taken from January 2012 to December 2015. Forty-one patients were hospitalized for acute headache and diagnosed with subarachnoid hemorrhage (SAH) by CT scan. Twenty-two patients with unruptured aneurysms were admitted for one or more of the following reasons: (1) aneurysms diagnosed on clinical investigation of headaches, transient ischemic attack, stroke, or seizures; (2) incidentally found on imaging; (3) incidentally found during hospitalization for another condition (e.g., head trauma); (4) signs and symptoms of cranial compression; or (5) sentinel headache (without evidence of SAH on CT or lumber puncture).

The patient exclusion criteria were: (1) malignant tumor; (2) malignant hypertension; or (3) any serious systemic disease. The aneurysm exclusion criteria were: (1) false aneurysm; (2) inflammatory aneurysm; (3) traumatic aneurysm; (4) dissecting aneurysm; or (5) aneurysm with arteriovenous malformation. The multiple aneurysms with unascertainable responsible aneurysms were also excluded.

The study protocol was approved by the ethics committee of the hospital. Informed consent was obtained from all patients. (Ethical approval number: 2016-28).

\section{Imaging}

All catheter angiograms were performed using standard transfemoral catheterization of the cerebral vessels, and digital subtraction angiography was performed using a Philips Integris Biplanar Unit (Philips Medical Systems, Best, The Netherlands). Rotational angiograms were obtained using a 6-s constant injection of contrast agent and a $180^{\circ}$ rotation with imaging at 15 frames/s for a total of $8 \mathrm{~s}$. Data from these images were transferred to the Philips Integris Workstation and reconstructed into 3D voxel data using the standard proprietary software provided with the system. 3D digital reconstruction that did not contain the entire aneurysm or associated parent arteries was excluded. The location of each aneurysm was recorded. Measurements of the aneurysm dome, neck, and the associated parent artery were performed using the conventional angiographic images and reference markers included in the view.

\section{Morphological assessments of aneurysms}

Two-dimensional (2D) variables were measured by digital subtraction angiography: height (the maximum distance from the center of the aneurysm neck and the dome of the aneurysm) and neck width (parallel to the parent artery). All aneurysms were divided into two categories: narrownecked (aspect ratio $\geq 1.4$ ) and wide-necked (aspect ratio $<1.4$ or neck width $\geq 4 \mathrm{~mm}$ ).

\section{Hemodynamic models}

Meshes were imported into the simulation software Fluent15 (ANSYS Inc., Canonsburg, PA, USA). The measured inlet flow rate was applied at the cervical segment of the internal carotid artery and far away from the aneurysm so that the flow within the aneurysm was not sensitive to the inflow boundary condition [18]. Blood was modeled as an incompressible Newtonian fluid with a density of $\rho=1050 \mathrm{~kg} / \mathrm{m}^{3}$ and a viscosity of $3.5 \times 10^{-3} \mathrm{~N} / \mathrm{m}^{2} \mathrm{~s}$. The governing equations were the unsteady state NavierStokes equation and the continuity equation in 3D. The given boundary conditions were the no-slip and rigid condition on the wall, flat velocity profile at the inlet, and zero pressure at the outlet. Patient-specific flow conditions were applied for the inlet, which were obtained postoperatively from transcranial Doppler measurement (mean flow 
rate of $185 \mathrm{ml} / \mathrm{min}$; maximum flow rate of $301 \mathrm{ml} / \mathrm{min}$; heart rate of $64 \mathrm{bpm}$; mean Reynolds number of 364; Womersley number of 2.32). The governing equations were solved using a finite volume method and by applying the SIMPLE method for pressure-velocity coupling $[19,20]$. Three pulsatile cycles were simulated to ensure that numeric stability had been reached, and the last cycle was taken as output.

\section{Hemodynamic parameter calculation}

WSS was calculated from the simulated flow fields of each aneurysm. The WSS for pulsatile flow was calculated by integrating the WSS magnitude at each node over the cardiac cycle (Eq. 1):

$\mathrm{WSS}=\frac{1}{T} \int_{0}^{T}\left|\mathrm{wss}_{i}\right| \mathrm{d} t$

The highest WSS (HWSS) was defined as the highest magnitude of intra-aneurysmal WSS. Mean WSS (MWSS) was the average of the WSS over the entire aneurysmal surface. Low WSS area ratio (LSAR) was defined as the area of the aneurysm wall exposed to a WSS below $10 \%$ of the mean parent arterial WSS normalized by the dome area [12].

Mean aneurysm-parent vessel WSS ratio (M-P WSS ratio): MWSS/PWSS.

Highest aneurysm-parent vessel WSS ratio (H-P WSS ratio): HWSS/PWSS.

PWSS (mean WSS of the near parent vessel) $(1.0 \mathrm{~cm}$ away from the aneurysm neck, Supplementary Fig. 1) in a cardiac cycle was measured in all 72 events. The MWSS and HWSS of the aneurysm and PWSS and the low WSS area (LSA) of the aneurysm were measured in all 72 events.

\section{Pathology}

Six wide-necked aneurysms (aspect ratio $<1.4$ or neck width $\geq 4 \mathrm{~mm}$ ) were resected, fixed, and paraffin-embedded after clipping. All narrow-necked aneurysms (aspect ratio $\geq 1.4$ ) were embolized using a Guglielmi detachable coil. The resected aneurysm wall was stained with hematoxylin-eosin and immunohistochemistry.

\section{Grouping}

Aneurysms were divided into two groups: ruptured and unruptured. Because of the close correlation between aspect ratio and hemodynamic factors, all aneurysms were divided into those with a narrow neck (aspect ratio $\geq 1.4$ ) and those with a wide neck (aspect ratio $<1.4$ or neck width $\geq 4 \mathrm{~mm}$ ). To eliminate the influence of hemodynamic factors due to different aspect ratios and to compare the true hemodynamic factors, the aneurysms were divided into four groups: (1) ruptured and narrow-neck; (2) unruptured and narrow-necked; (3) ruptured and widenecked; and (4) unruptured and wide-necked.

\section{Statistical analysis}

Statistical analysis was performed using SPSS 16.0 (IBM, Armonk, NY, USA). Correlation analyses were performed to explore how the geometrical morphology of the aneurysms affected hemodynamics. The Smirnov-Kolmogorov test was performed to determine the normality of the distribution of the continuous variables. Normally distributed continuous variables were expressed as means \pm standard deviations and analyzed using the independent-samples $t$ test. Non-normally distributed variables were presented as medians and quartiles and analyzed using the MannWhitney $U$ test. Two-sided $P$ values $<0.05$ were considered statistically significant.

Independent-sample $t$ tests and Mann-Whitney $U$ tests were performed between groups to confirm which hemodynamic factor was the most important index of aneurysm rupture. Binary logistic regression was performed separately using regression to assess the hemodynamic features that achieved univariate statistical significance $(P<0.05)$. Spearman correlation coefficients were performed between hemodynamic and geometrical morphology factors by bivariate correlation analysis.

\section{Results}

\section{Characteristics of the patients}

There were 21 men and 42 women. The patients were $34-82$ years of age (mean $56.9 \pm 9.43$ years).

\section{Characteristics of the aneurysms}

Three patients had two aneurysms, and three patients had three aneurysms. Of the selected aneurysms, 18 were located in the medial cerebral artery, 21 in the carotid artery, 26 in the posterior communicating artery, three in the anterior cerebral artery, and four in the ophthalmic artery. Anterior communicating artery aneurysms (which are visualized by two inflow injections with different directions) and vertebral basilary artery aneurysms with different diameter and blood flow distribution were excluded. Forty-one aneurysms were ruptured and 31 were unruptured. Aneurysm height ranged from 2.5 to $15.7 \mathrm{~mm}$, while the height-to-neck ratio ranged from 0.49 to 3.10 . 


\section{Correlations according to aneurysm rupture}

Ruptured aneurysms were more likely to have a greater LSAR $(P=0.001)$ and higher aneurysms parent WSS ratio $(P=0.026)$ than unruptured aneurysms (Table 1$)$. LSAR $(P=0.006)$ and the highest aneurysms parent WSS ratio $(P=0.023)$ were the hemodynamic factors predictive of rupture (Supplementary Table 1$)$. MWSS $(r=-0.558$, $P<0.001)$, mean aneurysm-artery WSS ratio $(r=-0.650, \quad P<0.001), \quad$ HWSS $\quad(r=-0.267$, $P=0.023)$, and the highest aneurysm-parent WSS ratio $(r=-0.342, P=0.003)$ were negatively correlated with aspect ratio. LSAR $(r=0.583, \mathrm{P}<0.001)$ was positively correlated with aspect ratio (Supplementary Fig. 2).

\section{Correlations according to aspect ratio}

Narrow-necked (aspect ratio $\geq 1.4$ ) aneurysms were more likely to have a larger LSAR $(P<0.001)$ and lower values of MWSS $(P<0.001)$, mean aneurysm-parent WSS ratio $(P<0.001)$, HWSS $(P=0.012)$, and the highest aneurysm-parent WSS ratio $(P<0.001)$ than wide-necked aneurysms (aspect ratio $<1.4$ or neck width $\geq 4 \mathrm{~mm}$ ) (Table 2).

\section{Correlations according to morphological categories}

In narrow-necked aneurysms, ruptured aneurysms were more likely to have larger $\operatorname{LSAR}(P=0.015)$ and lower values of MWSS $(P=0.028)$ and mean aneurysm-parent WSS ratio $(P=0.001)$ than unruptured aneurysms
(Table 3). Backward stepwise binary logistic regression indicated that mean aneurysm-parent WSS ratio $(P=0.018)$ was the only hemodynamic factor significantly predictive of rupture (Supplementary Table 2). In wide-necked aneurysms, ruptured aneurysms were more likely to have larger LSAR $(P=0.007)$, HWSS $(P=0.048)$, and the highest aneurysm-parent WSS ratio $(P<0.001)$ than unruptured aneurysms (Table 4$).$ LSAR and the highest aneurysm-parent WSS ratio (both $P=0.003)$ were the only hemodynamic factors predictive of rupture (Supplementary Table 3 ). On average, ruptured aneurysms seemed to have a greater area of lower WSS compared with the parent artery in narrow-necked aneurysms (Fig. 1), while ruptured aneurysms seemed to have a greater degree of WSS diversity and area of the higher WSS or lower WSS compared with the parent artery in wide-necked aneurysms (Fig. 1). The WSS of the surface area in unruptured aneurysms was not extreme (Fig. 2).

\section{Pathological examination}

The aneurysm wall showed two different types of pathological change after hematoxylin and eosin staining. The first type was characterized by a high number of degenerate smooth muscle cells (SMCs) producing collagen, with only sparse vascular endothelial cells and incomplete internal elastic lamina. No inflammatory response or atherosclerotic plaques were present in the high WSS aneurysms (Supplementary Fig. 3).

The second type was characterized by a large number of neutrophil granulocytes infiltrating the vessel wall, SMCs
Table 1 Wall shear stress and its ratios for ruptured and unruptured aneurysms

\begin{tabular}{llll}
\hline Parameters & Ruptured & Unruptured & $P$ \\
\hline Mean aneurysm WSS (Pa) & 3.15 & & \\
$\quad$ Median & 2.80 & 4.09 & 0.071 \\
Quartiles & & 2.05 & \\
Mean parent artery WSS (Pa) & 6.83 & 6.55 & 0.909 \\
Median & 6.27 & 4.37 & \\
Quartiles & 0.09 & & 0.001 \\
Low shear area ratio (low shear area/dome area) & 0.020 & \\
Median & 0.18 & 0.050 & 0.384 \\
Quartiles & 10.07 & 8.66 & \\
Highest aneurysm WSS (Pa) & 8.89 & 4.12 & 0.101 \\
Median & $0.511 \pm 0.293$ & $0.606 \pm 0.186$ & 0.026 \\
Quartiles & $1.439 \pm 0.421$ & $1.258 \pm 0.252$ & \\
Mean aneurysm-parent WSS ratio & & & \\
Highest aneurysm-parent WSS ratio &
\end{tabular}

Normally distributed variables are presented as mean \pm SD and were analyzed with the independentsamples $t$ test. Non-normally distributed variables are presented as medians and quartiles and were analyzed using the Mann-Whitney $U$ test. A $P$ value $<0.05$ was deemed statistically significant

WSS wall shear stress 
Table 2 Wall shear stress for narrow and wide-necked aneurysms

Table 3 Wall shear stress for ruptured and unruptured aneurysms in the narrow-necked aneurysm group

\begin{tabular}{llll}
\hline Parameters & Narrow & Wide & $P$ \\
\hline Mean aneurysm WSS (Pa) & & & $<0.001$ \\
$\quad$ Median & 2.37 & 4.33 & \\
Quartiles & 2.20 & 3.69 & 0.484 \\
Mean parent artery WSS (Pa) & 6.28 & 6.83 & \\
Median & 5.69 & 4.31 & $<0.001$ \\
Quartiles & 0.13 & & \\
Low shear area ratio (low shear area/dome area) & 0.30 & 0.020 & \\
Median & & & 0.078 \\
Quartiles & 7.58 & 9.23 & $<0.001$ \\
Highest aneurysm WSS (Pa) & 6.36 & 5.34 & $<0.001$ \\
Median & $0.327 \pm 0.181$ & $0.658 \pm 0.214$ & $1.470 \pm 0.309$ \\
Quartiles & $1.128 \pm 0.379$ & & \\
Mean aneurysm-parent WSS ratio & & & \\
Highest aneurysm-parent WSS ratio &
\end{tabular}

Normally distributed variables are presented as mean \pm SD and were analyzed with the independentsamples $t$ test. Non-normally distributed variables are presented as medians and quartiles and were analyzed using the Mann-Whitney $U$ test. A $P$ value $<0.05$ was deemed statistically significant

WSS wall shear stress

\begin{tabular}{lccc}
\hline Parameters & Ruptured & Unruptured & $P$ \\
\hline Mean aneurysms WSS (Pa) & $1.96 \pm 1.30$ & $3.33 \pm 1.45$ & 0.028 \\
Highest aneurysm WSS (Pa) & $9.08 \pm 6.64$ & $8.19 \pm 3.69$ & 0.720 \\
Mean parent artery WSS (Pa) & $7.85 \pm 3.98$ & $7.14 \pm 3.08$ & 0.655 \\
Low shear area ratio (low shear area/dome area) & $0.340 \pm 0.308$ & $0.093 \pm 0.125$ & 0.015 \\
Highest aneurysm-parent WSS ratio & $1.098 \pm 0.388$ & $1.174 \pm 0.382$ & 0.649 \\
Mean aneurysm-parent WSS ratio & $0.233 \pm 0.120$ & $0.474 \pm 0.164$ & 0.001 \\
\hline
\end{tabular}

Normally distributed variables are presented as mean \pm SD and were analyzed with the independentsamples $t$ test. A $P$ value $<0.05$ was deemed statistically significant

WSS wall shear stress

\begin{tabular}{llll}
\hline Parameters & Ruptured & Unruptured & $P$ \\
\hline Mean aneurysm WSS (Pa) & & & \\
$\quad$ Median & 4.04 & 4.44 & 0.587 \\
Quartiles & 5.24 & 2.96 & \\
Highest aneurysm WSS (Pa) & $14.58 \pm 10.93$ & $9.95 \pm 3.73$ & 0.048 \\
Mean parent artery WSS (Pa) & $8.94 \pm 6.13$ & $7.73 \pm 2.53$ & 0.355 \\
Low shear area ratio (low shear area/dome area) & $0.075 \pm 0.082$ & $0.026 \pm 0.032$ & 0.007 \\
Highest aneurysm-parent WSS ratio & $1.616 \pm 0.320$ & $1.292 \pm 0.176$ & 0.000 \\
Mean aneurysm-parent WSS ratio & $0.656 \pm 0.247$ & $0.660 \pm 0.170$ & 0.954 \\
\hline
\end{tabular}

Normally distributed variables are presented as mean \pm SD and were analyzed with the independentsamples $t$ test. Non-normally distributed variables are presented as medians and quartiles and were analyzed using the Mann-Whitney $U$ test. A $P$ value $<0.05$ was deemed statistically significant

WSS wall shear stress
Table 4 Wall shear stress for ruptured and unruptured aneurysms in wide-necked aneurysms thrombotic material in the low WSS aneurysms wall (Supplementary Fig. 4). Few vascular endothelial cells were observed after immunohistochemistry for CD31 in the with vacuoles and mucoid degeneration, hyperplasia of collagenous fibers in the matrix, internal elastic lamina, scarce vascular endothelial cells, and a large volume of 
Fig. 1 Top ruptured and narrow-neck aneurysm with a high ratio of low wall shear stress area. Middle and bottom ruptured and wide-neck aneurysm with a high ratio of low or highest wall shear stress area
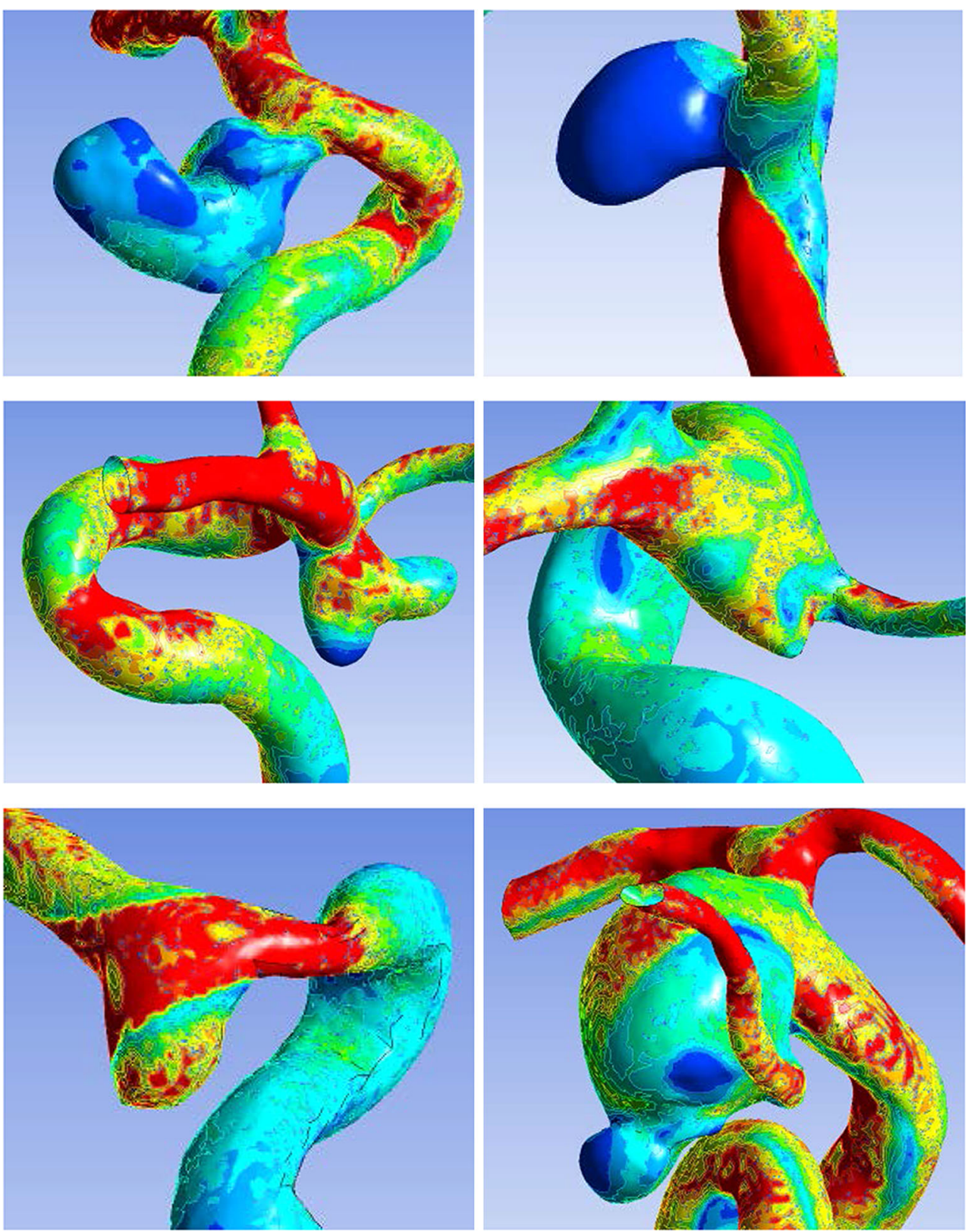

high WSS aneurysms (Supplementary Fig. 5), but a large number of neutrophil granulocytes infiltrating the vessel wall were observed after immunohistochemistry for CD68 in the low WSS aneurysms (Supplementary Fig. 6).

\section{Discussion}

Meng et al. [21] suggested that the "high-versus-low WSS" controversy is a manifestation of the complexity of aneurysm pathophysiology. Low WSS and high oscillatory shear index trigger an inflammatory cell-mediated pathway that could be associated with the growth and rupture of large, atherosclerotic aneurysms, while high WSS combined with a positive WSS gradient triggers a mural cell-mediated pathway that could be associated with the growth and rupture of small or secondary bleb aneurysm [21]. Yu et al. [22] showed that ruptured aneurysms showed higher mean WSS and maximum WSS than unruptured aneurysms. It is assumed that a WSS of $>2.0 \mathrm{~N} /$ $\mathrm{m}^{2}$ is suitable for maintaining the structure of the arterial vessels and that a WSS lower than $1.5 \mathrm{~N} / \mathrm{m}^{2}$ will lead to the degeneration of endothelial cells via apoptosis [23]. In the present study, the WSS in the LSA was obviously lower than $1.5 \mathrm{~N} / \mathrm{m}^{2}$. The highest aneurysm-parent WSS ratio was also a hemodynamic factors leading to aneurysm rupture, which is consistent with the study by Meng et al. [21]. 
Fig. 2 Top unruptured and narrow-neck aneurysm with a low ratio of low wall shear stress area. Bottom unruptured and wide-neck aneurysm with a low value of highest wall shear stress and no ratio of low wall shear stress area
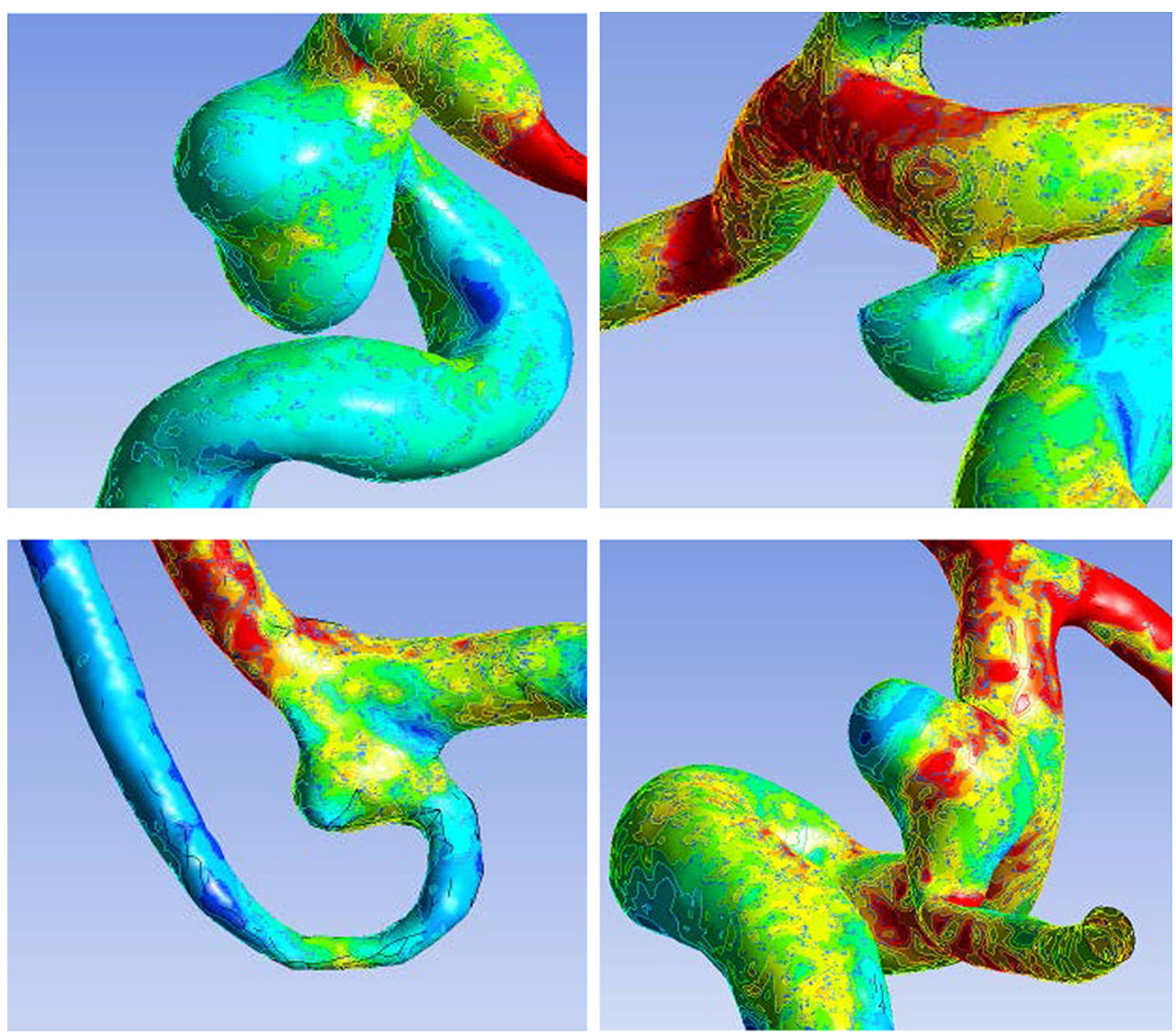

In clinical practice, aneurysmal geometry has been extensively used to estimate the likelihood of intracranial aneurysm rupture [24-27]. Amenta et al. [24] showed that an $\mathrm{AR}$ of 1.6, a dome diameter of $10 \mathrm{~mm}$, a deviated neck, and right-sidedness are independently associated with aneurysm rupture [24]. A study of multiple aneurysms in a single patient showed that aneurysms $>4 \mathrm{~mm}$ had a higher maximum WSS and area of low WSS, while aneurysms $<4 \mathrm{~mm}$ had a lower maximum WSS and area of low WSS [28]. There are many contradictory results in the literature. Aneurysm morphology itself has not been shown to lead to rupture. In the present study, hemodynamic variables were related to the geometric morphology of the aneurysm. A recent study showed that high flow impingement and WSS were associated with recanalization and regrowth, while low WSS was associated with aneurysm rupture [29].

Aspect ratio is negatively correlated with MWSS, mean aneurysm-artery WSS ratio, HWSS, and the highest aneurysm-parent WSS ratio, and positively correlated with LSAR. We found that aneurysms with an aspect ratio $<1.4$ or a neck width $\geq 4 \mathrm{~mm}$ (wide neck) had more inflow jet at the dome and a larger flow impingement region than aneurysms with an aspect ratio $\geq 1.4$. Aneurysms with an aspect ratio $<1.4$ or a neck width $\geq 4 \mathrm{~mm}$ had significantly higher MWSS, mean aneurysm-artery WSS ratio, HWSS, the highest aneurysm-parent WSS ratio, and less LSAR than aneurysms with an aneurysm with aspect ratio $\geq 1.4$.

The results of the present study strongly suggest that both high and low WSS were able to cause rupture in widenecked aneurysms. Occasionally distinct high and low WSS could be found in the same aneurysm. The highest aneurysm-parent WSS ratio and LSAR were the only differentiating factors in wide-necked aneurysms according to the binary logistic regression analyses. We assume that different parts of the intracranial vessel are unequally resistant to high WSS. Highest aneurysm-parent WSS ratio seemed to be a more sensitive factor than simple HWSS. Unruptured aneurysms with either wide or narrow necks had similar characteristics in that no distinct observations of high and low WSS were made.

According to Meng et al. [3], aneurysm lesion presentation is highly heterogeneous in almost every observable metric. The first type (type I) involves high WSS and a positive WSS gradient (spatial derivative of WSS along the flow direction with respect to the streamwise distance) $[21,30]$. Through endothelial cell mechanotransduction, these hemodynamic stresses initiate biochemical cascades when they exceed certain thresholds, leading to the local production and activation of proteases (the most important of which is matrix metalloproteinase) by wall cells [31], massive internal elastic lamina damage [30], and apoptosis 
[31], which are collectively responsible for media thinning and bulge formation [21]. Interestingly, inflammatory cell infiltration has not been observed in early stage intracranial aneurysm initiation [31].

In the second type (type II), the flow environment is likely to be dominated by low and oscillating WSS. This condition is exacerbated if secondary vortices form and/or flow instability increases [32]. Endothelial cells produce reactive oxygen species, up-regulate surface adhesion molecules and cytokines in the vessel wall, and increase luminal permeability $[33,34]$. These inflammatory infiltrates can produce large amounts of matrix metalloproteinases that degrade the extracellular matrix [35], thus tipping the balance between eutrophic and degradative processes and driving intracranial aneurysm growth and rupture [36]. Furthermore, such "disturbed flow" environments promote the formation of atherosclerotic plaques [36, 37]. The formation of a luminal thrombus can further trap macrophages and neutrophils and harbor proteases, reactive oxygen species, and oxidized low-density lipoproteins [38]. These two pathological types depend on the WSS distribution of the aneurysm. These differences indicate the close relationship between hemodynamic status and pathological change.

\section{Limitations}

Analysis of aneurysm shape to identify rupture factors relies on the hypothesis that aneurysm shape does not change after rupture. Even though increasing evidence indicates that such change does not occur [39], conclusive data on this subject remain scant. The pathological description of the aneurysms was made on only six samples. The vascular basement membrane and any extracellular matrix metalloproteinase were not visualized. There was the potential for some selection bias in the histological findings. Our CFD approach assumed rigid walls and did not take into account the viscoelasticity of the vessel wall because of the limited availability of physical information regarding arterial wall properties, such as elasticity and thickness. Finally, the affected artery was not taken into account in the analyses and a recent study suggested that the parameters affecting aneurysm rupture may depend on the artery harboring the aneurysm [40].

\section{Conclusion}

Aneurysm morphology could affect the distribution and magnitude of WSS on the basis of differences in blood flow. Both high and low WSS could contribute to focal wall damage and rupture through different mechanisms associated with each morphological type.
Acknowledgements This project was sponsored by Zhejiang provincial science and Technology Department of public welfare technology research social development project: Application study of computing fluid dynamics and morphology in intracranial aneurysm development, rupture and treatment (No. 2014C33SA600012).

\section{Compliance with ethical standards}

Funding This project was sponsored by Zhejiang provincial science and Technology Department of public welfare technology research social development project: Application study of computing fluid dynamics and morphology in intracranial aneurysm development, rupture and treatment (No. 2014C33SA600012).

Conflict of interest The authors declare that they have no conflict of interest.

Ethical approval All procedures performed in studies involving human participants were in accordance with the ethical standards of the research committee and with the 1964 Helsinki declaration and its later amendments or comparable ethical standards. The study protocol was approved by the ethics committee of Shaoxing People's Hospital (Ethical approval number: 2016-28).

Informed consent Informed consent was obtained from all individual participants included in the study.

Open Access This article is distributed under the terms of the Creative Commons Attribution 4.0 International License (http://crea tivecommons.org/licenses/by/4.0/), which permits unrestricted use, distribution, and reproduction in any medium, provided you give appropriate credit to the original author(s) and the source, provide a link to the Creative Commons license, and indicate if changes were made.

\section{References}

1. Hashimoto T, Meng H, Young WL (2006) Intracranial aneurysms: links among inflammation, hemodynamics and vascular remodeling. Neurol Res 28(4):372-380. doi:10.1179/016164106X14973

2. Xiang J, Natarajan SK, Tremmel M, Ma D, Mocco J, Hopkins LN, Siddiqui AH, Levy EI, Meng H (2011) Hemodynamicmorphologic discriminants for intracranial aneurysm rupture. Stroke J Cereb Circ 42(1):144-152. doi:10.1161/STROKEAHA. 110.592923

3. Meng H, Tutino VM, Xiang J, Siddiqui A (2014) High WSS or low WSS? Complex interactions of hemodynamics with intracranial aneurysm initiation, growth, and rupture: toward a unifying hypothesis. AJNR Am J Neuroradiol 35(7):1254-1262. doi:10.3174/ajnr.A3558

4. Kallmes DF (2012) Point: CFD-computational fluid dynamics or confounding factor dissemination. AJNR Am J Neuroradiol 33(3):395-396. doi:10.3174/ajnr.A2993

5. Cebral JR, Meng H (2012) Counterpoint: realizing the clinical utility of computational fluid dynamics-closing the gap. AJNR Am J Neuroradiol 33(3):396-398. doi:10.3174/ajnr.A2994

6. Robertson AM, Watton PN (2012) Computational fluid dynamics in aneurysm research: critical reflections, future directions. AJNR Am J Neuroradiol 33(6):992-995. doi:10.3174/ajnr.A3192

7. Cebral JR, Mut F, Weir J, Putman C (2011) Quantitative characterization of the hemodynamic environment in ruptured and 
unruptured brain aneurysms. AJNR Am J Neuroradiol 32(1):145-151. doi:10.3174/ajnr.A2419

8. Lu G, Huang L, Zhang XL, Wang SZ, Hong Y, Hu Z, Geng DY (2011) Influence of hemodynamic factors on rupture of intracranial aneurysms: patient-specific 3D mirror aneurysms model computational fluid dynamics simulation. AJNR Am J Neuroradiol 32(7):1255-1261. doi:10.3174/ajnr.A2461

9. Zhang Y, Mu S, Chen J, Wang S, Li H, Yu H, Jiang F, Yang X (2011) Hemodynamic analysis of intracranial aneurysms with daughter blebs. Eur Neurol 66(6):359-367. doi:10.1159/000332814

10. Castro M, Putman C, Radaelli A, Frangi A, Cebral J (2009) Hemodynamics and rupture of terminal cerebral aneurysms. Acad Radiol 16(10):1201-1207. doi:10.1016/j.acra.2009.03.022

11. Castro MA, Putman CM, Sheridan MJ, Cebral JR (2009) Hemodynamic patterns of anterior communicating artery aneurysms: a possible association with rupture. AJNR Am J Neuroradiol 30(2):297-302. doi:10.3174/ajnr.A1323

12. Jou LD, Lee DH, Morsi H, Mawad ME (2008) Wall shear stress on ruptured and unruptured intracranial aneurysms at the internal carotid artery. AJNR Am J Neuroradiol 29(9):1761-1767. doi:10. 3174/ajnr.A1180

13. Qian Y, Takao H, Umezu M, Murayama Y (2011) Risk analysis of unruptured aneurysms using computational fluid dynamics technology: preliminary results. AJNR Am J Neuroradiol 32(10):1948-1955. doi:10.3174/ajnr.A2655

14. Goubergrits L, Schaller J, Kertzscher U, van den Bruck N, Poethkow K, Petz C, Hege HC, Spuler A (2012) Statistical wall shear stress maps of ruptured and unruptured middle cerebral artery aneurysms. J R Soc Interface 9(69):677-688. doi:10.1098/rsif.2011.0490

15. Valencia A, Morales H, Rivera R, Bravo E, Galvez M (2008) Blood flow dynamics in patient-specific cerebral aneurysm models: the relationship between wall shear stress and aneurysm area index. Med Eng Phys 30(3):329-340. doi:10.1016/j.medengphy.2007.04.011

16. Schnell S, Ansari SA, Vakil P, Wasielewski M, Carr ML, Hurley MC, Bendok BR, Batjer H, Carroll TJ, Carr J, Markl M (2014) Three-dimensional hemodynamics in intracranial aneurysms: influence of size and morphology. J Magn Reson Imaging: JMRI 39(1):120-131. doi:10.1002/jmri.24110

17. Long Y, Yu H, Zhuo Z, Zhang Y, Wang Y, Yang X, Li H (2014) A geometric scaling model for assessing the impact of aneurysm size ratio on hemodynamic characteristics. Biomed Eng Online 13:17. doi:10.1186/1475-925X-13-17

18. Venugopal P, Valentino D, Schmitt H, Villablanca JP, Vinuela F, Duckwiler G (2007) Sensitivity of patient-specific numerical simulation of cerebal aneurysm hemodynamics to inflow boundary conditions. J Neurosurg 106(6):1051-1060. doi:10. 3171/jns.2007.106.6.1051

19. Shaffer N, Loth F (2012) CFD Challenge: modeling blood flow dynamics in a cerebral aneurysm using fluent. Paper presented at the ASME 2012 summer bioengineering conference, Puerto Rico

20. Irie K, Anzai H, Kojima M, Honjo N, Ohta M, Hirose Y, Negoro M (2012) Computational fluid dynamic analysis following recurrence of cerebral aneurysm after coil embolization. Asian J Neurosurg 7(3):109-115. doi:10.4103/1793-5482.103706

21. Meng H, Wang Z, Hoi Y, Gao L, Metaxa E, Swartz DD, Kolega J (2007) Complex hemodynamics at the apex of an arterial bifurcation induces vascular remodeling resembling cerebral aneurysm initiation. Stroke J Cereb Circ 38(6):1924-1931. doi:10. 1161/STROKEAHA.106.481234

22. Yu H, Li H, Liu J, Yang X (2016) An approach to quantitative assessment of hemodynamic differences between unruptured and ruptured ophthalmic artery aneurysms. Comput Methods Biomech Biomed Eng 19(13):1456-1461. doi:10.1080/10255842. 2016.1151009

23. Malek AM, Alper SL, Izumo S (1999) Hemodynamic shear stress and its role in atherosclerosis. JAMA 282(21):2035-2042
24. Amenta PS, Yadla S, Campbell PG, Maltenfort MG, Dey S, Ghosh S, Ali MS, Jallo JI, Tjoumakaris SI, Gonzalez LF, Dumont AS, Rosenwasser RH, Jabbour PM (2012) Analysis of nonmodifiable risk factors for intracranial aneurysm rupture in a large, retrospective cohort. Neurosurgery 70(3):693-699. doi:10.1227/ NEU.0b013e3182354d68 (discussion 699-701)

25. Ishibashi T, Murayama Y, Urashima M, Saguchi T, Ebara M, Arakawa H, Irie K, Takao H, Abe $\mathrm{T}$ (2009) Unruptured intracranial aneurysms: incidence of rupture and risk factors. Stroke J Cereb Circ 40(1):313-316. doi:10.1161/STROKEAHA. 108.521674

26. Lin N, Ho A, Gross BA, Pieper S, Frerichs KU, Day AL, Du R (2012) Differences in simple morphological variables in ruptured and unruptured middle cerebral artery aneurysms. J Neurosurg 117(5):913-919. doi:10.3171/2012.7.JNS111766

27. Qiu T, Jin G, Bao W (2014) The interrelated effects of 2D angiographic morphological variables and aneurysm rupture. Neurosciences 19(3):210-217

28. Jou L, Britz G (2016) Correlation between aneurysm size and hemodynamics in one individual with multiple small intracranial aneurysms. Cureus 8(7):e683. doi:10.7759/cureus.683

29. Liu J, Jing L, Wang C, Zhang Y, Yang X (2016) Recanalization, regrowth, and delayed rupture of a previously coiled unruptured anterior communicating artery aneurysm: a longitudinal hemodynamic analysis. World Neurosurg 89:726.e5-726.e10. doi:10. 1016/j.wneu.2016.01.002

30. Metaxa E, Tremmel M, Natarajan SK, Xiang J, Paluch RA, Mandelbaum M, Siddiqui AH, Kolega J, Mocco J, Meng H (2010) Characterization of critical hemodynamics contributing to aneurysmal remodeling at the basilar terminus in a rabbit model. Stroke J Cereb Circ 41(8):1774-1782. doi:10.1161/STRO KEAHA.110.585992

31. Kolega J, Gao L, Mandelbaum M, Mocco J, Siddiqui AH, Natarajan SK, Meng H (2011) Cellular and molecular responses of the basilar terminus to hemodynamics during intracranial aneurysm initiation in a rabbit model. J Vasc Res 48(5):429-442. doi: $10.1159 / 000324840$

32. Tremmel M, Dhar S, Levy EI, Mocco J, Meng H (2009) Influence of intracranial aneurysm-to-parent vessel size ratio on hemodynamics and implication for rupture: results from a virtual experimental study. Neurosurgery 64(4):622-630. doi:10.1227/ 01.NEU.0000341529.11231.69 (discussion 630-621)

33. Bian C, Xu G, Wang J, Ma J, Xiang M, Chen P (2009) Hypercholesterolaemic serum increases the permeability of endothelial cells through zonula occludens-1 with phosphatidylinositol 3-kinase signaling pathway. J Biomed Biotechnol 2009:814979. doi: $10.1155 / 2009 / 814979$

34. Ross R, Glomset JA (1976) The pathogenesis of atherosclerosis (first of two parts). N Engl J Med 295(7):369-377. doi:10.1056/ NEJM197608122950707

35. Galis ZS, Sukhova GK, Lark MW, Libby P (1994) Increased expression of matrix metalloproteinases and matrix degrading activity in vulnerable regions of human atherosclerotic plaques. J Clin Investig 94(6):2493-2503. doi:10.1172/JCI117619

36. Frosen J, Tulamo R, Paetau A, Laaksamo E, Korja M, Laakso A, Niemela M, Hernesniemi J (2012) Saccular intracranial aneurysm: pathology and mechanisms. Acta Neuropathol 123(6):773-786. doi:10.1007/s00401-011-0939-3

37. Chiu JJ, Chien S (2011) Effects of disturbed flow on vascular endothelium: pathophysiological basis and clinical perspectives. Physiol Rev 91(1):327-387. doi:10.1152/physrev.00047.2009

38. Ross R (1999) Atherosclerosis-an inflammatory disease. N Engl J Med 340(2):115-126. doi:10.1056/NEJM199901143400207

39. Rahman M, Ogilvy CS, Zipfel GJ, Derdeyn CP, Siddiqui AH, Bulsara KR, Kim LJ, Riina HA, Mocco J, Hoh BL (2011) Unruptured cerebral aneurysms do not shrink when they rupture: 
multicenter collaborative aneurysm study group. Neurosurgery 68(1):155-160. doi:10.1227/NEU.0b013e3181ff357c (discussion 160-151)

40. Duan G, Lv N, Yin J, Xu J, Hong B, Xu Y, Liu J, Huang Q (2016) Morphological and hemodynamic analysis of posterior communicating artery aneurysms prone to rupture: a matched case-control study. J Neurointerv Surg 8(1):47-51. doi:10.1136/ neurintsurg-2014-011450 